\title{
Ruptured ectopic in a spontaneous heterotopic gestation; management and spontaneous vertex delivery of a live baby at term.
}

\author{
Nonye-Enyidah Esther Ijeoma* and Sekibo Boma \\ Department of Obstetrics and Gynaecology, Rivers State University Teaching Hospital, Port Harcourt.
}

Publication history: Received on 07 January 2020; revised on 12 January 2020; accepted on 20 January 2020

Article DOI: https://doi.org/10.30574/wjarr.2020.5.1.0008

\begin{abstract}
Heterotopic pregnancy is the simultaneous co-existence of an intrauterine and extrauterine pregnancy. It is uncommon and potentially dangerous occurring in 1:30,000 of natural conceptions. It carries a significant maternal morbidity and mortality including foetal wastage due to the risk of ectopic gestation. To report a case of ruptured ectopic in a spontaneous heterotopic gestation at 8 weeks of amenorrhoea. The case note of the patient was retrieved and the management reviewed. Relevant review of literature on the subject was also done. She was a 25 year old nullipara who presented at the accident and emergency unit of Rivers State University Teaching Hospital at 8 weeks gestation with two weeks history of lower abdominal pain with no associated bleeding per vaginam. There was haemoperitoneum from a ruptured tubal pregnancy co-existing with live intrauterine pregnancy diagnosed on ultrasound scan. She had emergency exploratory laparotomy and right partial salpingectomy. Intraoperative findings were haemoperitoneum of 1.5 litres, bulky uterus and right ruptured isthmic ectopic gestation. She did well post operatively and was discharged home to continue with antenatal care. She subsequently delivered a live male baby that weighed $3.4 \mathrm{~kg}$ at term. Mother and baby were discharged in good clinical state. Clinicians must be alert to the fact that confirming an intrauterine pregnancy clinically or by ultrasound scan does not exclude the co-existence of an ectopic gestation. A high index of suspicion is needed for early diagnosis of heterotopic pregnancy even in the absence of risk factors; to prevent maternal mortality and have a favourable obstetric outcome.
\end{abstract}

Keywords: Heterotopic pregnancy; Laparotomy; Partial salpingectomy; Ruptured ectopic

\section{Introduction}

Heterotopic pregnancy (HP) is a rare and dangerous life-threatening condition that is difficult to diagnose and easily missed. It usually occurs in women at risk of ectopic pregnancy and those undergoing fertility treatments [1-3]. It occurs in 1: 30,000 spontaneous pregnancies and 1: 3,900 pregnancies following Assisted Reproductive Techniques (ART) and ovulation induction [4-6]. Pregnancies following ART have an estimated 1\% chance of resulting in HP [7-9] Therefore it should be suspected in any patient who present with lower abdominal pain especially without vaginal bleeding in a pregnancy resulting from fertility treatment [7-9]. Other risk factors are pelvic inflammatory disease (P.I.D), tubal damage, pelvic surgery, uterine mullerian abnormalities, tubal surgery, previous ectopic pregnancy and tubo-ovarian abscess. The location of the ectopic gestation sac can be at the fallopian tube, uterine cornua, cervix or in the previous caesarean section scar $[10,11]$.

Heterotopic pregnancy is commonly diagnosed at the time of rupture necessitating surgical management [1-3]. Diagnosis is difficult but a significant discrepancy between B-HCG levels and the corresponding intrauterine pregnancy can point to a possibility of another pregnancy. They usually present with abdominal pain especially without vaginal bleeding, peritoneal irritation and bulky uterus which can be larger than the gestational age. They may also present with hypovolaemic shock or asymptomatic in some cases [3-5]. Early symptoms can also be similar to those seen in acute

\footnotetext{
${ }^{*}$ Corresponding author

E-mail address: hernsi@yahoo.com
} 
appendicitis or accidented ovarian cyst [3-5]. Transvaginal ultrasound scan plays an important role in the diagnosis of $\mathrm{HP}$ which presents as an intrauterine pregnancy co-existing with a separate adnexal mass, gestational sac or ring sign $[12,13]$.

Surgery is the key to the management of HP. The goal is to remove the ectopic pregnancy without jeopardizing the intrauterine pregnancy $[1,2,14,15]$. Laparoscopic salpingectomy is the standard surgical approach [16]. Other management options include expectant management, laparotomy and salpingectomy, local injection of potassium chloride and hyperosmolar glucose under ultrasound guidance followed by aspiration of the ectopic gestation $[1,2,3$, $14,15]$. A literature review found survival rate of $64.4 \%$ of the intrauterine foetus and miscarriage can occur in $35.6 \%$ [16].

\section{Case report}

Mrs. B.R was a 25 year old business woman, Gravida 4 Para $0^{+3}$ with secondary level of education. She presented at the accident and emergency unit of the hospital at 8 weeks gestation with 2 weeks history of progressively increasing lower abdominal pain. Pain was sudden in onset, initially mild, intermittent and dull; localized in the right iliac fossa. Pain radiated to the right shoulder tip, worsened on lying down.There were no histories of trauma to the abdomen, fall, bleeding per vaginam and urinary symptoms. There was history of vomiting prior to presentation and she had an episode of fainting spell. She neither had fertility treatment nor previous pelvic surgery. For the above complaints she presented to a general hospital where pregnancy was confirmed by serum B-HCG and was given an analgesic which temporarily relieved the pain. She was subsequently referred to Rivers State University Teaching Hospital (RSUTH). Pregnancy was spontaneously achieved.

On examination, she was in severe painful and respiratory distress, afebrile $\left(37.1^{\circ} \mathrm{C}\right)$, pale, anicteric and there was no pitting pedal oedema. Her chest was clinically clear but respiratory rate was 27 beats/min.Her pulse rate was $116 \mathrm{bpm}$ and blood pressure was 100/80 $\mathrm{mmHg}$. Her abdomen was distended. There were generalized tenderness, guarding, and rebound tenderness. Pointing sign was negative. Bowel sounds were present and normoactive. Vulva and vagina were normal, uterus was bulky and cervix was firm, long and cervical os was closed. Pouch of Douglas was full and cervical motion tenderness wad positive. A transvaginal ultrasound scan which she presented with revealed a viable intrauterine pregnancy with a right ruptured ectopic gestation and a moderate haemoperitoneum. No other abnormality was detected A diagnosis of ruptured ectopic in a spontaneous heterotopic gestation was made.

She was admitted. Urgent packed cell volume was $16 \%$, retroviral screening for HIV $1 \& 2$ was sero-negative, white blood cell count was $8000 / \mathrm{uL}$ and urinalysis was normal. Four units of blood were grouped and cross matched. She was placed on nothing by mouth and one litre of normal saline was set up; parenteral antibiotics and analgesics were also given. Urethral catheter was passed and retained. She was informed of the diagnosis and counseled on the need for an emergency exploratory laparotomy. Informed and written consent were obtained. Theatre was booked and anaesthetists were informed.

She subsequently had an emergency exploratory laparotomy and right partial salpingectomy with minimal handling of the uterus. Intra operative findings were haemoperitoneum of 1.5 litres, bulky uterus, right ruptured isthmic ectopic gestation, normal left tube and ovaries. She was transfused with 2 units of blood intra operatively. Post operatively, she was continued on the intravenous fluid, parenteral antibiotics, analgesics and salbutamol for 48 hours and was converted to oral drugs. She also received 2 more units of blood. Her packed cell volume on the second day after surgery was $30 \%$. Post operative period was unremarkable and she was discharged home on the 5 th post operative day. She was also advised to register for antenatal care which she did. The antenatal care subsequently remained uneventful until she had a spontaneous vaginal delivery of a live male baby who weighed $3.4 \mathrm{~kg}$ at term. Both mother and baby were discharged home in good clinical state.

\section{Discussion}

Early diagnosis of HP is challenging because of lack of clinical signs and symptoms as well as diagnostic confusion in early pregnancy [1-4]. A good history as was done in this case is important to rule out the risk factors of HP. The patient had terminated three pregnancies although she claimed there were no post abortal complications. If there is intrauterine pregnancy with abdominal pain, a full work up and close monitoring should be done to rule out HP especially following assisted reproduction technology (ART) techniques [8,9]. The pregnancy in this case was spontaneously conceived. In the presence of haemoperitoneum in a confirmed intrauterine pregnancy, it is important to consider the possibility of a ruptured heterotopic gestation as was seen in this case presented. Other misleading 
presentations of HP include threatened or incomplete miscarriage. In about $50 \%$ of cases, HP can remain asymptomatic till its discovery by a routine first trimester ultrasound scan $[1,4,5]$. Fortunately in this case, both intrauterine and extrauterine pregnancies were discovered simultaneously by ultrasound scan.

A review article that analyzed six articles in 2008 [13] showed that transvaginal ultrasonography has a sensitivity of $74-84 \%$ in diagnosing ectopic gestation and specificity of $84-99.9 \%$. However, it is operator dependent. The only pathognomonic sign of HP is the visualization of extrauterine and intrauterine fetal poles with cardiac motion as in our patient. This occurs in $10 \%$ of cases [13]. A feature on ultrasound scan associated with ectopic pregnancies which was discovered in our case was haemoperitoneum due to bleeding from the ruptured and damaged tube. This should have made the sonologist to do a thorough inspection of the adnexae despite observing an intrauterine pregnancy. Also ectopic gestation normally occurs on the ipsilateral side of the corpus luteum in $70-85 \%$ of cases [13].

The management of HP is challenging because one needs to preserve the viable intrauterine pregnancy as well as resolving the ectopic gestation [1-3]. Treatment of HP can be surgical, medical or expectant. The procedure is usually salpingectomy via laparotomy or laparoscopy depending on patient's haemodynamic status at the time of diagnosis. Depending on the state of the contralateral fallopian tube or the patient's reproductive needs and the site of the ectopic gestation, it is possible to consider conservation of the affected tube $[1,2,4,5]$. In our case, emergency laparotomy and partial salpingectomy with minimal handling of the uterus was carried out because of the haemodynamic state of the patient at presentation. Preserving the life of the mother and the intrauterine foetus was the aim of management of this HP and this was achieved by shortest time under general anaesthesia, minimal handling of the uterus during surgery, blood transfusion and use of salbutamol to relax the uterus after surgery. The management also allowed the viable intrauterine pregnancy to develop to term and she subsequently had a spontaneous vaginal delivery.

\section{Conclusion}

A high index of suspicion for heterotopic pregnancy is advocated in early pregnancy with abdominal pain and signs and symptoms of ectopic gestation especially haemoperitoneum. Ultrasonography plays a vital role in the early diagnosis of heterotopic pregnancy thereby preventing maternal morbidity and mortality caused by this dangerous obstetric condition.

\section{Compliance with ethical standards}

\section{Acknowledgments}

We wish to express our profound gratitude to everybody who participated in the management of this patient.

\section{Disclosure of conflict of interest}

Authors have declared that no competing interests exist.

\section{References}

[1] ChadeeA, Rezal S, Kirby C, Chadwick E et al. (2016). Spontaneous heterotopic pregnancy: Dual case report and review of literature. Case reports in Obstetrics and Gynaecology, 10, 1-5.

[2] Headley AJ andAdom V. (2013). Naturally occurring heterotopic pregnancy in a multiparous patient: case report. Journal of Reproductive of Medicine, 58 (11-12), 541-544.

[3] Fatema N, Al Badi MM, Rahman M andElawdy MM. (2016). Heterotopic pregnancy with natural conception: a rare that is still being misdiagnosed: a case report. Clinical case reports, 4 (3), 272-275.

[4] Altintas E, Yuksel B, Tok S et al. (2015). "Heterotopic pregnancy identified in the post partum period". International journal of Gynaecology and Obstetrics, 130 (3), 287-288.

[5] Martin JK and Gala RB. (2015). Adnexal mass in a spontaneous pregnancy diagnosed as heterotopic pregnancy at the time of caesarean delivery. Fall, 15 (3), 265-267.

[6] Nargund A, Majumdar S and Stokes I. (2013). "Heterotopic pregnancy after spontaneous conception". Journal of Obstetrics and Gynaecology, 33(4), 425. 
[7] Rafaat B, Dalton E and Ledger WL. (2015). Ectopic pregnancy secondary to in vitro fertilization-embryo transfer; pathogenic mechanisms and management strategies. Reproductive Biology and Endocrinology, 13 (1), 30.

[8] Buca DI, Margano D, Impicciatore G et al. (2015). Early diagnosis of heterotopic triplet pregnancy with an intrauterine and bilateral tubal pregnancy after IVF: a case report. Journal of Obstetrics and Gynaecology, 35, 755-756.

[9] Perkins KM, Boulet SL, Kissin DM et al. (2015). Risk of ectopic pregnancy associated with assisted reproductive technology in the United State, 2001-2011. Obstet.Gynecol, 125, 70-78.

[10] Tingi E, Rowland J and Hanna L. (2014). A case of heterotopic pregnancy following spontaneous conception. Journal of Obstetrics and Gynaecology, 35 (4), 430-431.

[11] Talbot K, Simpson R, Price N et al. (2014). Misdiagnosis and delayed diagnosis for ectopic and heterotopic pregnancies after in vitro fertilization and embryo transfer. Journal of Huazhorg University of Science, Technology and Medical Science, 34, 103-107.

[12] Kwok TC and Morgan G. (2012). Think heterotopic: A case report of heterotopic pregnancy detected on through ultrasonography. Journal of Medical Cases, 3 (5), 326-328.

[13] Bignardi T, Alhamadan D andCondous G. (2008). Is ultrasound the new gold standard for the diagnosis of ectopic pregnancy? Semin ultrasound CT MR, 29 (2), 114-120.

[14] Jin-Bo LI, Ling-Zhi K, Jian-Bo Y et al. (2016). Management of heterotopic pregnancy: experience from 1 tertiary medical centre. Medicine, 95 (5), e2570.

[15] Marion LL and Meeks GR. (2012). Ectopic pregnancy: history, incidence, epidemiology and risk factors. Clinical Obstetrics and Gynaecology, 55, 376-386.

[16] Fouedjio JH, Fouelifack FY et al. (2013). Ruptured heterotopic pregnancy in a natural conception cycle: a case report at the Yaounde Central Hospital (Cameroon). The Pan African Medical Journal, 16 (106), 3506.

\section{How to cite this article}

Nonye-Enyidah EI and Sekibo B. (2020). Ruptured ectopic in a spontaneous heterotopic gestation; management and spontaneous vertex delivery of a live baby at term. World Journal of Advanced Research and Reviews, 5(1), 112-115. 